\title{
Modus Operandi: Irrigation of the Modified Eloesser Flap in Heterogeneous Suppurative Lung Pathologies
}

\author{
Rajkamal Vishnu, M.Ch. ${ }^{,}$Guruprasad D. Rai, M.Ch. ${ }^{1}$, Ganesh Sevagur Kamath, M.Ch. ', Vijaya Kumara, M.D. ${ }^{2}$ \\ Departments of 'Cardiovascular and Thoracic Surgery and ${ }^{2}$ Anesthesia, Kasturba Medical College, Manipal Academy of Higher Education, Manipal, India
}

\section{ARTICLE INFO}

Received November 5, 2020

Revised December 24, 2020

Accepted January 25, 2021

Corresponding author

Guruprasad D. Rai

Tel $91-9886730726$

Fax 91-9886730726

E-mail guru.rai@manipal.edu

ORCID

https://orcid.org/0000-0002-8785-0803

\begin{abstract}
Background: Refractory empyemas with collapsed lung and persistent bronchopleural fistulas pose significant problems to thoracic surgeons and impose a substantial burden in terms of morbidity and mortality. The modified Eloesser flap procedure is a useful palliative option for clearing infections. Herein, we present our experiences with the modified Eloesser flap procedure in mixed suppurative lung pathologies with a new technique of irrigation for persistent infection.

Methods: A retrospective review was carried out of 56 patients who underwent the modified Eloesser flap with continuous irrigation at Katurba Medical College. These patients had severe morbidities and were not suitable for major thoracic resection surgery, and electively underwent modified Eloesser flap surgery. Regular follow-up was done at 1 , 3,6 , and 12 months. Patients with persistent infections were treated with our continuous irrigation technique.

Results: The most important finding was that all patients with active sputum acid-fast bacilli-positive findings became sputum smear-negative during the first month of follow-up. Half (50\%) of the patients had a patent stoma. Eleven patients had persistent infections, necessitating continuous irrigation. The infection was fully cleared after 1 month in 9 patients, while 2 patients required second irrigation and continued to receive follow-up. In the remaining $50 \%$ of the patients, the stoma closed completely, and the lung expanded fully.

Conclusion: The modified Eloesser flap is a simple procedure. In suppurative pathologies, infections were well controlled and the general condition of the patients improved. Our continuous irrigation method showed promising results in patients with persistent purulent discharge.
\end{abstract}

Keywords: Modified Eloesser flap, Lung disease, Therapeutic irrigation, Thoracic empyemas tuberculosis, Povidone-iodine

\section{Introduction}

The management of refractory empyemas with collapsed lung and persistent bronchopleural fistulas (BPF) poses a significant problem to thoracic surgeons. In India, tuberculosis (TB) is the most prevalent cause of empyema and causes considerable morbidity and mortality. Parapneumonic effusions are the commonest cause of refractory empyema, while other causes (e.g., post-lung resection surgery, trauma, etc.) are less frequently identified [1]. These patients have poor lung function, chronic infection, and malnutrition, which make them unfit for major thoracic surgery. Furthermore, these patients are initially treated with antibiotics, tube thoracostomy, and thrombolysis, but the infection sometimes does not resolve. For these patients, Eloesser developed a procedure that is a useful palliative option for clearing the infection [2]. Later, the modified Eloesser flap (MEF) developed by Symbas et al. [3] emerged as a successful alternative for treatment of empyema, BPF [4], and post-surgical space infection. Many other techniques for pleural obliteration once the infection is under control, after the MEF procedure, have been described [5,6]. Herein, we describe our experiences of the MEF procedure in mixed suppurative lung pathologies 
with a new technique of irrigation for persistent infections and present the results.

\section{Methods}

Data collection was carried out through a retrospective review of 56 consecutive patients undergoing a MEF at Kasturba Medical College, Manipal, India. The observational study period was 5 years, from January 2012 to January 2017. Institutional ethical approval was obtained, and the study was conducted according to the institutional ethical protocol. Patients undergoing MEF in our study had various suppurative lung pathologies such as chronic empyema, BPF, and post-lung resection space infection. There were $44(78.5 \%)$ male patients and $12(21.4 \%)$ female patients. Their mean age was 42.3 years (range, $21-69$ years).

Our study subjects were routinely investigated for persistent lung pathologies and underwent conventional chest roentgenograms and standard laboratory evaluations. Sputum was obtained for acid-fast bacilli (AFB) staining in all the patients, of whom 9 (17.85\%) had multi-drug-resistant pulmonary tuberculosis (MDR-TB) and underwent the conventional MDR-TB regimen as per the Revised National TB Control Programme, India prior to surgery (Table 1). Preoperative pulmonary function testing, chest computed tomography (CT), and fiber-optic bronchoscopy scanning were performed prior to surgery. A screening of patients' history for diabetes, prior pulmonary TB, and cardiac illness was done. Most of our patients were referred from the Department of Pulmonary Medicine after multiple rounds of failed medical treatment, and others were old postopera-

Table 1. Preoperative patient status, tuberculosis category, and treatment

\begin{tabular}{lr}
\hline \multicolumn{1}{c}{ Variable } & \multicolumn{1}{c}{ No. $(\%)$} \\
\hline Total no. of patients in the study group & $56(100.00)$ \\
Total no. of pulmonary tuberculosis patients & $20(35.71)$ \\
Patients on anti-tubercular drugs prior to MEF ${ }^{\mathrm{a}, \mathrm{b})}$ & $20(35.71)$ \\
Sputum-positive at the time of MEF & $9(16.07)$ \\
Sputum-negative at the time of MEF & $11(19.64)$ \\
Total no. of MDR-TB patients & $9(16.07)$ \\
\hline
\end{tabular}

MEF, modified Eloesser flap; MDR, multi-drug-resistant; TB, tuberculosis; RNTCP, Revised National TB Control Programme.

a) For new cases of pulmonary tuberculosis, the treatment regimen as per RNTCP included 2 months of isoniazid, rifampicin, ethambutol, and pyrazinamide in the intensive phase and 4 months of isoniazid, rifampicin, and ethambutol in the continuation phase. ${ }^{\text {b) The MDR-TB }}$ treatment regimen as per RNTCP included 6-9 months of kanamycin, levofloxacin, ethionamide, cycloserine, pyrazinamide, and ethambutol in the intensive phase and 18 months of levofloxacin, ethionamide, cycloserine, and ethambutol in the continuation phase. tive lung resection patients (these patients are previously operated long time ago) requiring MEF for space infection. Common therapeutic modalities that were used prior to MEF included image-guided catheter placement, thrombolytic administration, intercostal drain (ICD) placement, and decortication.

In our study, all the patients electively underwent MEF. The surgical technique of MEF followed the description of Symbas et al. [3]. The patients were put in the right or left lateral position depending on the side of MEF to be done. All the patients underwent a uniform U-shaped inverted skin incision which was decided after needle localization on the table, scars of a previous intercostal tube, or thoracotomy. The flap was fashioned at the basal region of the thorax. A window was placed near the diaphragm for maximum gravity-dependent drainage when the patient sat in an upright or semi-upright position. After fashioning the skin and subcutaneous tissue, deeper planes were dissected using electrocautery in the same inverted U-shaped flap until adequate width and length were achieved. Together, it had a tongue-like appearance, and was later used to create the window. The rib to be resected depended on the extent of drainage required. Once the pleura was opened, surgical debridement of non-viable tissue was done and tissue samples were collected for cultures. A thorough wash was performed to clear all the infective foci. Once again, the adequacy of the window was checked before creating the stoma. The soft tissue flap was now re-fashioned inward against the diaphragm on the most dependent position and the skin edges of the upper region were not sutured to the pleural surface. The wound was inspected for hemostasis and a moist dressing with paraffin gauge was placed. Most of the patients required 2-3 rounds of dressing with low thoracic suction to remove all the retained collection with saline irrigation (except in a patient with BPF) for faster recovery [4]. All patients were educated about wound care at home and regular dressing.

Patients with persistent purulent discharge were treated with a continuous irrigation technique. These patients were admitted, and routine cultures and blood investigations were performed. For the irrigation process, a $12 \mathrm{~F}$ pigtail catheter was placed at the second anterior intercoastal space at midclavicular line with ultrasound-guided catheter insertion by the Seldinger technique under local anesthesia. To avoid fluid spillage and to allow easy maintenance of the window flap, a colostomy bag was placed at the window site. Continuous irrigation was carried out twice daily for 5 days. Irrigation was first performed with $200 \mathrm{~mL}$ of $1 \%$ povidone-iodine solution over a period of 60 
minutes, followed by a 1-L saline flush over a period of 20 minutes. Patients were then encouraged to change positions frequently to maintain a uniform distribution. The collected fluid was disposed along with the colostomy bag and a moist dressing with a paraffin gauge was applied. The same procedure was repeated. Meanwhile, antibiotics were started according to the culture results and sensitivity.

From the collected data, information was extracted on patients' age, sex, the indications for surgery, past medical history, the presence of active $\mathrm{TB}$, preoperative investigations, intraoperative procedures, length of postoperative stay, microorganism culture reports, status of stoma patency, complications of the procedure, and postoperative follow-up.

Observational data were entered from patients' charts and analyzed using SPSS ver. 15.0 (SPSS Inc., Chicago, IL, USA).

The study was approved by the Institutional Review Board of Kasturba Medical College (IRB approval no., IEC 415-2020). Informed consent was obtained from all individual participants included in the study.

\section{Results}

In our study, 44 men and 12 women were admitted with mixed suppurative lung pathology. The most common conditions requiring the MEF that we encountered were chronic empyema (30 patients, 53.5\%), post-lung resection space infection (10 patients, $17.8 \%$ ), BPFs after lung surgery (6 patients, 10.7\%), trapped lung (4 patients, $7.14 \%$ ), and chronic empyema with BPF (6 patients, 10.7\%), as detailed in (Fig. 1). All the patients were routinely investigated with basic blood analyses, sputum samples for AFB, chest roent-

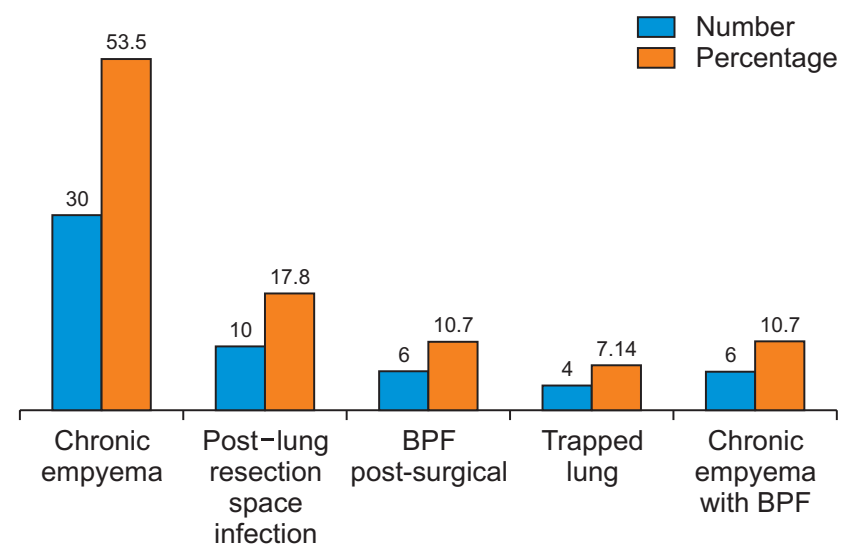

Fig. 1. Heterogeneity of infections. BPF, bronchopleural fistula. genograms, chest CT, and intraoperative fluid and tissue cultures before undergoing the MEF procedure. The most common organisms from intraoperative fluid and tissue cultures were Gram-positive organisms (mainly Staphylococcus and Streptococcus) and Gram-negative organisms (mainly Pseudomonas) (Fig. 2). Eighteen patients were diagnosed with diabetes mellitus, but fortunately had well-controlled blood sugar levels. Every patient had ICD in situ for a relatively long period with failure of lung expansion and other medical management. Fifty-six patients electively underwent MEF. The mean time period from disease onset to MEF was $8 \pm 4.42$ weeks. The operative technique was the same for all the patients, using U-shaped inverted flap. The intraoperative and in-hospital outcomes are presented in Table 2. Adequate intraoperative drainage was achieved in patients with space infection and empyema, and there were no perioperative complications or deaths. In all patients with diabetes mellitus, the MEF

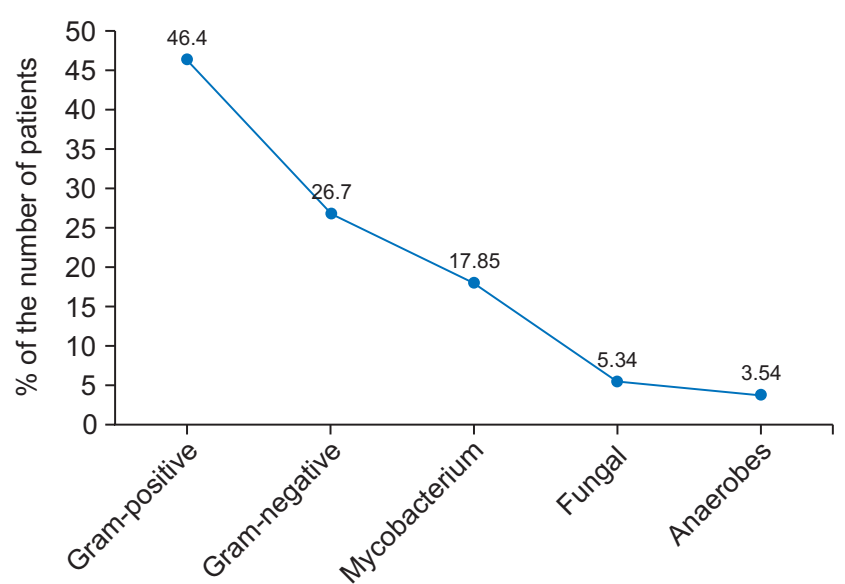

Fig. 2. Types of micro-organisms. Values are presented as \% of the number of patients.

Table 2. Intraoperative and postoperative outcomes of patients undergoing the modified Eloesser flap and our irrigation method

\begin{tabular}{lc}
\hline \multicolumn{1}{c}{ Outcomes } & Value \\
\hline Inverted U-flap & $100(56)$ \\
In-hospital mortality & 0 \\
Mean postoperative stay (day) & $8.73(6-13)$ \\
Follow-up status & \\
$\quad$ Stoma patent & $50(28)$ \\
Stoma closed & $50(28)$ \\
Mean stoma closure time (mo) & $7 \pm 4.2$ \\
Continuous irrigation success rate (first time) & $81(9)$ \\
Continuous irrigation success rate (second time) & $100(2)$ \\
\hline
\end{tabular}

Values are presented as \% (number), mean (range), or mean \pm standard deviation. 

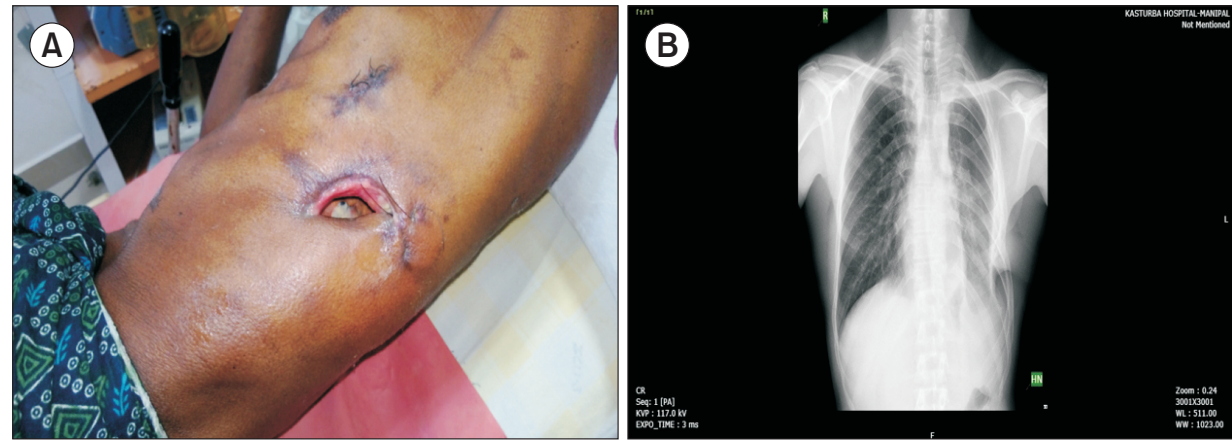

Fig. 3. (A) Postoperative follow-up after 1 month, showing a wellhealed stoma. (B) Postoperative chest X-ray with an expanded lung on the left side.

showed favorable granulation and healing, which was achieved with adequate blood glucose control (Fig. 3A, B). Regular follow-up was done at 1, 3, 6, and 12 months postoperatively, and 2 deaths occurred during follow-up due to respiratory arrest with sepsis and cachexia. Most of our patients clinically improved with weight gain and increased appetite after MEF. Due to continuous gravity-dependent drainage and the absence of stasis of purulent fluid, the infection was controlled well in most of the patients during the first month of follow-up. In 28 patients (50\%) who underwent MEF, the window was completely closed, of whom $92.9 \%$ (26) had complete lung expansion and 2 (7.1\%) required enlargement of the window for drainage as the air leak persisted. The mean closure period was $7 \pm 4.20$ months. In the remaining 28 patients (50\%), the window was patent; of these patients, 17 (60.7\%) had complete lung expansion and 11 (39.2\%) had persistent infections and continued to receive follow-up. The 11 patients with persistent infections (of whom 9 patients had MDR-TB and 2 patients had Gram-positive bacterial infections) were treated with our continuous irrigation technique described above (Fig. $4 \mathrm{~A}, \mathrm{~B})$. During the first month of follow-up, out of $11 \mathrm{pa}-$ tients who underwent continuous irrigation with a patent window, 9 patients showed no infective discharge and their lungs had fully expanded (7 patients with MDR-TB converted to sputum smear-negative and 2 patients with Grampositive bacteria showed negative culture results) and continued to receive regular follow-up. Two patients with MDRTB with persistent infections were treated for a second time with continuous irrigation after 1 month, and continued to receive follow-up with no further purulent discharge and negative sputum smear findings.

\section{Discussion}

Many different techniques for window drainage have been developed with modifications for a few decades. The
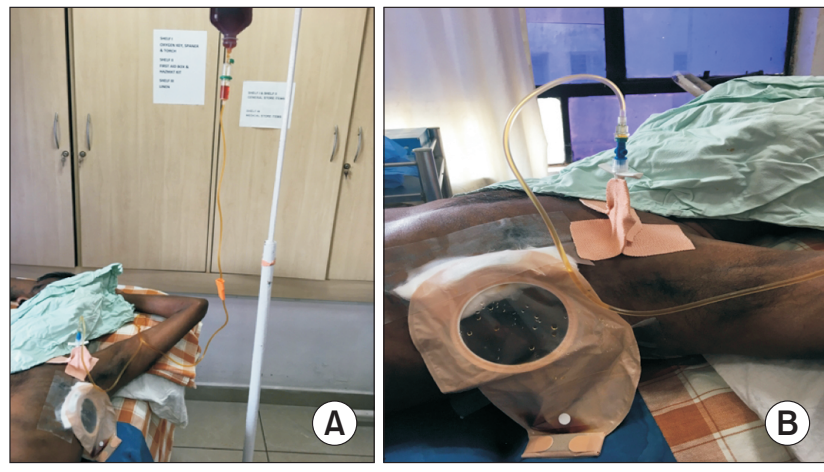

Fig. 4. (A, B) Continuous irrigation method with a pigtail catheter and colostomy bag for collection of drainage.

original window drainage procedure was introduced by Eloesser [2] was developed for draining tuberculous empyemas and pleural space infections. His original technique involved a superior U-shaped flap over the most dependent position with resection of the underlying ribs. His drainage method acted as a 1-way valve where purulent content exits and air is prevented from re-entering the pleural space. The valve also provides negative pressure, which encourages lung expansion. This method was further modified by Symbas et al. [3] in 1971 for the treatment of thoracic empyema. The modified technique consists of an inverted U-shaped flap at the basal region of the thorax with resection of the ribs. Once the cavity is totally drained and the underlying ribs resected, the tongue flap is then tacked to the inferior-most aspect of the drained space. In recent years, the MEF has been a very effective drainage technique for the treatment of thoracic empyema [7].

In our current study, we adopted the MEF using an inverted $U$-shaped flap in all patients [3]. Our indications for surgery extended beyond chronic empyema to include BPF and post-resection space infections. Prior to surgery, all patients underwent nonsurgical treatments such as antibiotics, multiple thoracentesis, and intercostal tube place- 
ment $[1,7]$. The reasons for performing this procedure included anticipation of prolonged intercostal tube placement and non-compliance with intercostal tube placement and position. In this study, we observed that an active pulmonary TB patient improved with MEF, and as a result of the continuous irrigation method with povidone-iodine, all MDR-TB patients converted to sputum smear AFB-negative status during follow-up [8]. Overall, in our patients, who had serious morbidities and were not suitable for major thoracic resection surgery, MEF served as a palliative procedure improving quality of life [7]. To highlight the advantages of MEF in our patients: (1) it is a minor procedure well tolerated by patients with severe morbidities; (2) aggressive diabetes control promoted a faster recovery with healthy granulation tissue; (3) infection control was achieved in most of our patients with symptomatic improvement (reduction in purulent discharge, increase in appetite, and weight gain) and enhanced overall quality of life; (4) BPF recovered quickly with lung expansion, changing into a bronchocutaneous fistula [4]; and (5) the chest deformity after spontaneous stoma closure was very minimal and the residual defect was a small indentation on the skin at the site of the stoma. For patients with a persistent stomal opening, thoracoplasty has been advised as an option. During follow-up, 11 patients had persistent infections that were treated with continuous irrigation. This technique was previously used for post-pneumonectomy space infections.

The advantages of our continuous irrigation method are that: (1) a thorough cleaning of the purulent substance is possible; (2) it is less painful, as a $12 \mathrm{~F}$ pigtail catheter is used under local anesthesia; (3) lung injury is avoided, as the catheter is inserted using ultrasound guidance; (4) using a colostomy bag has additional benefits for avoiding spillage of contents and easy maintenance of the MEF stoma; (5) the use of only $1 \%$ povidone-iodine is non-toxic to the lung and leads to better healing [9,10]; and (6) all MDRTB patients showed negative sputum smear results during follow-up after undergoing our continuous irrigation technique $[11,12]$.

In conclusion, the modified Eloesser type of drainage is a simple and safe procedure to perform. Prompt clearance of infection is seen after the procedure. In most cases, it is sufficient for the closure of BPF. In cases where fistula closure did not occur, the infection was well controlled. Additionally, our continuous irrigation technique had the following advantages: (1) it helped to resolve persistent infections; (2) it was very convenient for both surgeons and patients; and (3) adequate infection control was observed in this irrigation technique, as it was a continuous method. The general condition of patients improved, providing the opportunity to undertake a more extensive procedure at a later date at a considerably lower risk. This technique is highly valuable for patients who do not understand their disease and who are not compliant with instructions pertaining to the care of a long-standing ICD. The residual chest deformity after window closure is acceptable. This study shows how an alternative, less-invasive method of irrigation can prevent recurrent lung infection and improve quality of life.

Our study is probably the first one to describe this technique and hence has limitations as a retrospective observational study with no prior studies to compare. We also acknowledge that the small sample size may not provide adequate statistical power to the study, thereby limiting the extrapolation of the study results to the general population.

\section{Conflict of interest}

No potential conflict of interest relevant to this article was reported.

\section{ORCID}

Rajkamal Vishnu: https://orcid.org/0000-0002-0294-6196 Guruprasad D. Rai: https://orcid.org/0000-0002-8785-0803 Ganesh Sevagur Kamath: https://orcid.org/0000-0003-1020-2717 Vijaya Kumara: https://orcid.org/0000-0003-4642-7213

\section{References}

1. Magovern CJ, Rusch VW. Parapneumonic and post-traumatic pleural space infections. Chest Surg Clin N Am 1994;4:561-82.

2. Eloesser L. Of an operation for tuberculous empyema. Ann Thorac Surg 1969;8:355-7.

3. Symbas PN, Nugent JT, Abbott OA, Logan WD Jr, Hatcher CR Jr. Nontuberculous pleural empyema in adults: the role of a modified Eloesser procedure in its management. Ann Thorac Surg 1971;12:6978.

4. Galvin IF, Gibbons JR, Maghout MH. Bronchopleural fistula: a novel type of window thoracostomy. J Thorac Cardiovasc Surg 1988;96: 433-5.

5. Clagett OT, Geraci JE. A procedure for the management of postpneumonectomy empyema. J Thorac Cardiovasc Surg 1963;45:141-5.

6. Gharagozloo F, Margolis M, Facktor M, Tempesta B, Najam F. Postpneumonectomy and postlobectomy empyema. Thorac Surg Clin 2006;16:215-22.

7. Thourani VH, Lancaster RT, Mansour KA, Miller JI Jr. Twenty-six 
years of experience with the modified Eloesser flap. Ann Thorac Surg 2003;76:401-5.

8. Subotic D, Yablonskiy P, Sulis G, et al. Surgery and pleuro-pulmonary tuberculosis: a scientific literature review. J Thorac Dis 2016;8: E474-85.

9. Gharagozloo F, Trachiotis G, Wolfe A, DuBree KJ, Cox JL. Pleural space irrigation and modified Clagett procedure for the treatment of early postpneumonectomy empyema. J Thorac Cardiovasc Surg 1998;116:943-8
10. Banerjee A, Subbarao KS. Use of povidone iodine for the management of post-pneumonectomy empyema. Chest 1983;84:507-8.

11. Rikimaru T, Kondo M, Kajimura K, et al. Efficacy of common antiseptics against multidrug-resistant mycobacterium tuberculosis. Int $\mathrm{J}$ Tuberc Lung Dis 2002;6:763-70.

12. Rikimaru T, Kondo M, Kajimura K, et al. Bactericidal activities of commonly used antiseptics against multidrug-resistant mycobacterium tuberculosis. Dermatology 2002;204 Suppl 1:15-20. 